\title{
Inflammatory Macrophages in the Sciatic Nerves Facilitate Neuropathic Pain Associated with Type 2 Diabetes Mellitus ${ }^{[\mathrm{s}}$
}

\author{
Fumihiro Saika, ${ }^{1}$ @ Norikazu Kiguchi, ${ }^{1}$ @Shinsuke Matsuzaki, ${ }^{1}$ @Daichi Kobayashi, \\ and Shiroh Kishioka
}

Department of Pharmacology, Wakayama Medical University, Wakayama, Japan

Received August 5, 2018; accepted December 31, 2018

\begin{abstract}
Despite the requirement for effective medication against neuropathic pain associated with type 2 diabetes mellitus (T2DM), mechanism-based pharmacotherapy has yet to be established. Given that long-lasting neuroinflammation, driven by inflammatory macrophages in the peripheral nerves, plays a pivotal role in intractable pain, it is important to determine whether inflammatory macrophages contribute to neuropathic pain associated with T2DM. To generate an experimental model of T2DM, C57BL/6J mice were fed a high-fat diet (HFD) ad libitum. Compared with control diet feeding, obesity and hyperglycemia were observed after HFD feeding, and the mechanical pain threshold evaluated using the von Frey test was found to be decreased, indicating the development of mechanical allodynia. The expression of mRNA markers for macrophages, inflammatory cytokines, and chemokines were significantly upregulated in the sciatic nerve (SCN) after HFD feeding. Perineural administration of
\end{abstract}

saporin-conjugated anti-Mac1 antibody (Mac1-Sap) improved HFD-induced mechanical allodynia. Moreover, treatment of Mac1-Sap decreased the accumulation of $\mathrm{F} 4 / 80^{+}$macrophages and the upregulation of inflammatory mediators in the SCN after HFD feeding. Inoculation of lipopolysaccharide-activated peritoneal macrophages in tissue surrounding the SCN elicited mechanical allodynia. Furthermore, pharmacological inhibition of inflammatory macrophages by either perineural or systemic administration of TC-2559 [4-(5-ethoxy-3-pyridinyl)- $N$-methyl(3E)-3-buten-1-amine difumarate], a $\alpha 4 \beta 2$ nicotinic acetylcholine receptor-selective agonist, relieved HFD-induced mechanical allodynia. Taken together, inflammatory macrophages that accumulate in the SCN mediate the pathophysiology of neuropathic pain associated with T2DM. Inhibitory agents for macrophagedriven neuroinflammation could be potential candidates for novel pharmacotherapy against intractable neuropathic pain.

\section{Introduction}

Type 2 diabetes mellitus (T2DM) is a chronic metabolic disease and results in various long-term complications affecting the eyes, kidneys, and peripheral nervous system. Approximately $50 \%$ of patients with T2DM experience painful neuropathy (neuropathic pain) (Boulton et al., 2005; Sudore et al., 2012) that is resistant to currently available medications such as opioid analgesics (Barrett et al., 2007). Despite the high prevalence and negative impact on quality of life, the molecular basis of the pathogenesis of neuropathic pain associated with T2DM is yet to be elucidated. Therefore, there is a need to clarify the key components underlying T2DMrelated neuropathic pain to establish novel effective pharmacotherapies. Among the variety of basic approaches used to

This work was supported by Smoking Research Foundation and Japan Society for the Promotion of Science KAKENHI Grant Number JP16K08994.

${ }^{1}$ F.S., N.K., and S.M. contributed equally to this work.

https://doi.org/10.1124/jpet.118.252668.

S This article has supplemental material available at jpet.aspetjournals.org. uncover the pathogenesis of complications in T2DM, high-fat $\operatorname{diet}(\mathrm{HFD}$ )-induced diabetes with obesity has proved to be the most popular experimental model in rodents, being closely correlated with the known pathology of T2DM (Heydemann, 2016). For example, T2DM experimental rodent models induced by HFD feeding and patients with T2DM present hyperexcitability and spontaneous activity of primary sensory neurons reflecting the typical symptoms of neuropathic pain, such as hyperalgesia and allodynia (Baron et al., 2009; Menichella et al., 2014; Jayaraj et al., 2018). Therefore, HFD feeding is also a useful approach to investigate the molecular background of T2DM-related neuropathic pain in rodents.

Emerging evidence suggests that chronic neuroinflammation due to neuroimmune interactions plays a pivotal role in the pathogenesis of intractable neurogenic disorders, such as autoimmune diseases, neurodegenerative diseases, and neuropathic pain (Ubogu et al., 2006; Scholz and Woolf, 2007; Drouin-Ouellet and Cicchetti, 2012). In addition, numerous reports have demonstrated that the infiltration of circulating leukocytes (i.e., monocytes/macrophages and neutrophils) to

ABBREVIATIONS: ACTB, $\beta$-actin; CCL, CC-chemokine ligand; CD, control diet; CD11b, cluster of differentiation molecule 11b; CD68, cluster of differentiation 68; $\mathrm{DH} \beta \mathrm{E}$, dihydro- $\beta$-erythroidine hydrobromide $[(2 \mathrm{~S}, 13 \mathrm{bS})-2$-methoxy-2,3,5,6,8,9,10,13-octahydro-1H,12H-benzo[i]pyrano[3,4g]indolizin-12-one hydrobromide]; DMEM, Dulbecco's modified Eagle's medium; HFD, high-fat diet; IL, interleukin; IRF5, interferon regulatory factor 5; LPS, lipopolysaccharide; Mac1-Sap, saporin-conjugated anti-Mac1 antibody; nAChR, nicotinic acetylcholine receptor; PBS, phosphate-buffered saline; PBST, phosphate-buffered saline containing 0.3\% Triton X-100; qPCR, quantitative polymerase chain reaction; RT, reverse transcription; SCN, sciatic nerve; TC-2559, 4-(5-ethoxy-3-pyridinyl)- $N$-methyl-(3E)-3-buten-1-amine difumarate; T2DM, type 2 diabetes mellitus; TLR4, toll-like receptor 4; TNF $\alpha$, tumor necrosis factor $\alpha$. 
the damaged nervous system contributes significantly to chronic neuroinflammation (Liu et al., 2000; Hu and McLachlan, 2002; Thacker et al., 2007; Austin and Moalem-Taylor, 2010; Calvo et al., 2012). Particularly, macrophages account for the largest population among infiltrating leukocytes in injured peripheral nerves, and the depletion of macrophages clearly prevents experimental neuropathic pain in rodents (Liu et al., 2000; Mert et al., 2009; Kobayashi et al., 2015). Moreover, it has been reported that several inflammatory cytokines [e.g., interleukin (IL) $-1 \beta$ and tumor necrosis factor $\alpha(\mathrm{TNF} \alpha)$ ] and chemokines [e.g., CC-chemokine ligand (CCL) 3 and CCL4], derived from inflammatory macrophages, exert functions as key neuroinflammatory regulators that directly enhance the excitability of primary sensory neurons and prolong neuroinflammation, facilitating neuropathic pain (Sommer and Kress, 2004; Scholz and Woolf, 2007; Lee and Zhang, 2012; Saika et al., 2012). Given the functional significance of peripheral inflammatory macrophages in the pathogenesis of neuropathic pain, it is worth investigating the pathophysiological roles of such macrophages in T2DM model mice.

The primary aim of this study was to determine whether inflammatory macrophage numbers increased in the peripheral nervous system of the T2DM model mice after HFD feeding and whether inflammatory macrophages contribute to the pathogenesis of T2DM-related neuropathic pain by applying inhibitors of inflammatory macrophages. According to our previous reports, the $\alpha 4 \beta 2$ nicotinic acetylcholine receptor ( $\mathrm{nAChR}$ ) ligand TC-2559 difumarate [4-(5-ethoxy3 -pyridinyl)- $N$-methyl-(3E)-3-buten-1-amine difumarate] is a potent inhibitor of inflammatory macrophages and improved neuropathic pain after peripheral nerve injury in mice (Kiguchi et al., 2015b; Saika et al., 2015). We also evaluated the therapeutic potential of TC-2559 administration targeting peripheral macrophages for T2DM-related neuropathic pain. Such investigations not only provide scientific understanding regarding the pathophysiology of T2DM, but also suggest novel evidence-based pharmacotherapy focusing on peripheral macrophages for intractable neuropathic pain associated with T2DM.

\section{Materials and Methods}

Generation of T2DM Mice. All animal experiments were approved by the Animal Research Committees of Wakayama Medical University and were carried out in accordance with the in-house guidelines for the care and use of laboratory animals of Wakayama Medical University. Male C57BL/6J mice, 8 weeks of age (SLC, Hamamatsu, Japan), were used in all experiments, and experiments complied with the Ethical Guidelines of the International Association for the Study of Pain. Mice were housed in plastic cages in a temperature-controlled room $\left(23-24^{\circ} \mathrm{C}, 60 \%-70 \%\right.$ humidity) with a 12-hour dark/light cycle and provided with water and food ad libitum. To induce T2DM and control, mice were fed an HFD (60 kcal\% fat; catalog number D12492; Research Diets Inc., New Brunswick, NJ) or a control diet (CD; catalog number D12450J; Research Diets Inc.), respectively, ad libitum. For the measurement of blood glucose levels, a small incision was made in the tail of the mice. Then approximately $5 \mu \mathrm{l}$ of whole blood was collected, and blood glucose was analyzed using GLUCOCARD with dia-sensor (Arkray Inc., Tokyo, Japan).

Drug Administration. Saporin-conjugated anti-Mac1 (CD11b) antibody (Mac1-Sap) and control saporin were purchased from Advanced Targeting Systems (San Diego, CA). TC-2559 and dihydro- $\beta$-erythroidine hydrobromide ( $\mathrm{DH} \beta \mathrm{E})[(2 \mathrm{~S}, 13 \mathrm{bS})-2$-methoxy$2,3,5,6,8,9,10,13$-octahydro- $1 \mathrm{H}, 12 \mathrm{H}$-benzo[i]pyrano[3,4-g]indolizin12-one hydrobromide] were purchased from Tocris Biosciences (Bristol, UK). These agents were dissolved in sterile phosphatebuffered saline (PBS) and administered by systemic (subcutaneous) or perineural injection according to a previously described method (Ma and Quirion, 2006; Maeda et al., 2009). In brief, under isoflurane anesthesia, either agent $(10 \mu \mathrm{l})$ was injected without a skin incision into the region surrounding the sciatic nerve (SCN), using a microsyringe fitted with a 30 -gauge needle connected to a cannula. Injections of Mac1-Sap or control saporin were administered three times every 2 days. On the other hand, TC-2559 and $\mathrm{DH} \beta \mathrm{E}$ were administered consecutively for 4 days after 16 weeks of HFD feeding.

Macrophage Culture and Inoculation. Under isoflurane anesthesia, an incision was made in the peritoneal membrane of naive mice, and $3 \mathrm{ml}$ of chilled sterile PBS containing $1 \%$ penicillinstreptomycin was slowly injected into the peritoneal cavity. After flushing, collected peritoneal macrophages were washed with PBS and red blood cells were lysed by incubating for 1 minute with $150 \mathrm{mM}$ $\mathrm{NH}_{4} \mathrm{Cl}, 10 \mathrm{mM} \mathrm{KHCO} 3$ and $1 \mathrm{mM}$ EDTA containing buffer at room temperature. The cells were collected by centrifugation at $400 \times \mathrm{g}$ for 5 minutes, then cultured in Dulbecco's modified Eagle's medium (DMEM; Sigma-Aldrich, Tokyo, Japan) containing 1\% penicillinstreptomycin at $37^{\circ} \mathrm{C}$ in an atmosphere of $5 \% \mathrm{CO}_{2}$. The cells were incubated with lipopolysaccharide (LPS; Sigma-Aldrich) dissolved in DMEM for 3 hours. Subsequently, $8 \times 10^{4}$ cells of LPS-exposed macrophages were resuspended in $10 \mu \mathrm{l}$ of DMEM and perineurally administered to another mouse in a volume of $10 \mu \mathrm{l}$.

Behavioral Testing. For evaluating mechanical allodynia, the $50 \%$ withdrawal threshold was determined by the von Frey test in accordance with a previously established method (Chaplan et al., 1994). Briefly, mice were individually placed on a $5 \times 5 \mathrm{~mm}$ wire mesh grid floor and covered with an opaque acrylic box. After adaptation for 2-3 hours, calibrated von Frey filaments (Neuroscience, Tokyo, Japan) were applied to the middle of the plantar surface of the hind paw through the bottom of the mesh floor. In the paradigm of the up-down method, testing was initiated with a $0.4 \mathrm{~g}$ force in the middle of the series $(0.02,0.04,0.07,0.16,0.4,0.6,1.0,1.4$, and $2.0 g)$. Stimuli were always presented in a consecutive fashion, either ascending or descending. In the absence of a paw withdrawal response to the selected force, a stronger stimulus was applied. In the presence of paw withdrawal, the next weaker stimulus was chosen. In accordance with the findings by Chaplan et al. (1994), after the response threshold was first crossed (the two responses straddling the threshold), four additional stimuli were applied. Based on the responses to the series of the von Frey filament, the $50 \%$ paw withdrawal threshold was calculated.

Whole-Mount Immunohistochemistry. The SCN was collected from mice after transcardiac perfusion with PBS followed by $4 \%$ paraformaldehyde and was postfixed in $4 \%$ paraformaldehyde at $4{ }^{\circ} \mathrm{C}$ overnight. The SCN was treated with PBS containing $0.3 \%$ Triton X-100 (PBST) for 1 hour and then blocked with 5\% normal donkey serum in $0.3 \%$ PBST at room temperature for 2 hours. The SCN was then incubated with primary antibodies against F4/80 (rat monoclonal, 1:200; Cederlane, Burlington, ON, Canada) and the $\alpha 4$ subunit of the nAChR (rabbit polyclonal, 1:300; Alomone Labs, Jerusalem, Israel) at $4^{\circ} \mathrm{C}$ overnight. Antibodies were diluted in $1 \%$ normal donkey serum in $0.1 \%$ PBST. The following day, the SCN was rinsed in PBST and incubated with fluorescence-conjugated secondary antibodies (1:300; Abcam, Cambridge, MA) at room temperature for 2 hours. The SCN was rinsed in PBST, and then incubated with Hoechst 33342 stain (Thermo Fisher Scientific, Waltham, MA) at room temperature for 10 minutes. Finally, the SCN was mounted on cover glass, and fluorescence images were immediately acquired using a confocal laser scanning microscope (Carl Zeiss, Oberkochen, Germany). For the quantification of macrophages, the number of $\mathrm{F} 4 / 80^{+}$cells was measured in a square with an area of $100 \times 100 \mu \mathrm{m}^{2}$ 
and was shown as the average of three randomly selected sections from one SCN of each mouse.

Reverse Transcription-Quantitative Polymerase Chain Reaction. Mice were euthanized by decapitation, and the fresh SCNs and trigeminal nerves were collected in RNAlater solution (Thermo Fisher Scientific). The TRIzol Plus RNA Purification Kit (Thermo Fisher Scientific) was used for the isolation of total RNA from the tissues following the manufacturer instructions. The SCN was placed in a 1.5-ml RNase-free tube and homogenized with TRIzol reagent. Chloroform was added to each sample, which was then centrifuged at $4^{\circ} \mathrm{C}$ for 15 minutes. The aqueous phase containing RNA was transferred to a fresh tube, and RNA was isolated by purification column. Total RNA extract was used for the synthesis of cDNA by reverse transcription (RT) as follows. Total RNA was incubated with Random Primers (Promega, Madison, WI) at $70^{\circ} \mathrm{C}$ for 5 minutes and then cooled on ice. We then transcribed cDNA by incubation with M-MLV Reverse Transcriptase (Promega) and dNTP mix (Promega) at $37^{\circ} \mathrm{C}$ for 50 minutes. Quantitative polymerase chain reaction ( $\mathrm{qPCR}$ ) was performed using the AriaMx RealTime PCR System (Agilent Technologies, Santa Clara, CA) with template cDNA, primers for each gene (Thermo Fisher Scientific) and SYBR Premix Ex Taq II (Takara Bio Inc., Kusatsu, Japan). The primer sequences are listed in Table 1 . Reactions were performed under the following conditions: 3 minutes at $95^{\circ} \mathrm{C}$, followed by 45 cycles of two steps, 10 seconds at $95^{\circ} \mathrm{C}$ and 30 seconds at $60^{\circ} \mathrm{C}$. The fluorescence intensities were recorded, and data were normalized to $\beta$-actin (ACTB).

Statistical Analysis. Data are presented as the mean \pm S.E.M. Statistical analyses were performed using Student's $t$ test, one-way analysis of variance followed by Tukey's multiple-comparison test, or two-way analysis of variance followed by Bonferroni's multiplecomparison test as appropriate. Statistical significance was established at $P<0.05$.

\section{Results}

We first generated the T2DM model mice by HFD feeding ad libitum. The body weight of the HFD group was significantly greater than that of the CD group during the whole experimental period $[\mathrm{F}(1,126)=1246.27, P<0.0001$; Bonferroni's test, $P<0.001 ; 4-24$ weeks; $n=10$ ] (Fig. 1A). The mice exhibited obesity at 16 weeks of HFD feeding compared with CD feeding (Fig. 1B). Blood glucose levels after 4 weeks of HFD feeding were higher than those for CD feeding, and hyperglycemia persisted for at least 24 weeks of feeding $[\mathrm{F}(1,91)=123.17, P<0.0001$; Bonferroni's test, $P<0.05$; 4-24 weeks; $n=5-10$ ] (Fig. 1C). The mechanical pain threshold evaluated by the von Frey test was significantly decreased at 8 weeks in contrast to the decrease of at least
24 weeks of HFD feeding, indicating long-lasting mechanical allodynia $[\mathrm{F}(1,100)=75.23, P<0.0001$; Bonferroni's test, $P<0.01 ; 8-24$ weeks; $n=8-10$ ] (Fig. 1D).

Next, we investigated macrophage accumulation and the expression of inflammatory mediators in the SCN by RT-qPCR. The mRNA expression levels of inflammatory macrophage markers $(\mathrm{F} 4 / 80[\mathrm{~F}(1,30)=44.94, P<0.0001$; Bonferroni's test, $P<0.001 ; n=6], \mathrm{CD} 11 \mathrm{~b}[\mathrm{~F}(1,30)=51.70$, $P<0.0001$; Bonferroni's test, $P<0.001 ; n=6$ ], cluster of differentiation 68 (CD68) $[\mathrm{F}(1,30)=45.08, P<0.0001$; Bonferroni's test, $P<0.001 ; n=6]$, and IRF5 $[\mathrm{F}(1,30)=20.79$, $P<0.0001$; Bonferroni's test, $P<0.01 ; n=6$ ]) were upregulated at 16 weeks of HFD feeding in contrast to that after CD feeding (Fig. 2A). Consistently, pain-related inflammatory cytokines $(\mathrm{IL}-1 \beta[\mathrm{F}(1,30)=19.93, P=0.0001$; Bonferroni's test, $P<0.01 ; n=6]$ and $\mathrm{TNF} \alpha[\mathrm{F}(1,30)=9.17, P=0.0050$; Bonferroni's test, $P<0.05 ; n=6]$ ) and chemokines (CCL3 $[\mathrm{F}(1,30)=43.95, P<0.0001$; Bonferroni's test, $P<0.001$; $n=6]$ and CCL4 $[\mathrm{F}(1,30)=19.22, P=0.0001$; Bonferroni's test, $P<0.05 ; n=6]$ ) were also upregulated at 16 weeks of HFD feeding (Fig. 2B). These expression patterns persisted for 32 weeks of HFD feeding, suggesting the accumulation of inflammatory macrophages and long-lasting neuroinflammation in the SCN. On the other hand, upregulation of neither macrophage markers nor inflammatory cytokines and chemokines was observed in the trigeminal nerves at 16 weeks of HFD feeding (Supplemental Fig. 1).

To determine the contribution of peripheral macrophages to HFD-induced mechanical allodynia, Mac1-Sap (macrophagetargeting toxin, $250 \mathrm{ng})$ or control saporin $(250 \mathrm{ng})$ was perineurally administered three times, every 2 days after 16 weeks of HFD feeding. HFD-induced allodynia was significantly improved on day 7 after the first Mac1-Sap treatment $[\mathrm{F}(3,72)=33.02, P<0.0001$; Bonferroni's test, $P<0.05 ; n=9-11]$ and had no effect in the CD group. Control saporin did not affect the mechanical pain threshold in either the CD or HFD group (Fig. 3A). We confirmed that F4/80 macrophages were accumulated in the SCN after 17 weeks of HFD feeding, and the number of $\mathrm{F} 4 / 80^{+}$macrophages decreased after Mac1-Sap treatment, as determined by immunohistochemistry $[\mathrm{F}(3,12)=23.77, P<0.0001$; Tukey's test, $P<0.001 ; n=4$ ] (Fig. 3B).

We then examined whether the ablation of inflammatory macrophages affects the expression of inflammatory mediators in the SCN. The mRNA expression levels of F4/80 ( $t$ test, $P=0.0066 ; n=6)$ and CD11b ( $t$ test, $P=0.0306 ; n=6)$ at

TABLE 1

Primer sequences for RT-qPCR

\begin{tabular}{lll}
\hline \multicolumn{1}{c}{ Gene } & \multicolumn{1}{c}{ Forward $\left(5^{\prime}\right.$ to $\left.3^{\prime}\right)$} & Reverse $\left(5^{\prime}\right.$ to $\left.3^{\prime}\right)$ \\
\hline GAPDH & GGGTGTGAACCACGAGAAAT & ACTGTGGTCATGAGCCCTTC \\
ACTB & CAGCTGAGAGGGAAATCGTG & TCTCCAGGGAGGAAGAGGAT \\
F4/80 & AACTTTCAAGGCCCAGGAGT & GCTCTCCCCAGGATATTGGT \\
CD11b & GTTTCTACTGTCCCCCAGCA & GTTGGAGCCGAACAAATAGC \\
CD68 & ACTCATAACCCTGCCACCAC & CCAACAGTGGAGGATCTTGG \\
IRF5 & ACACTGAAGGGGTGGATGAG & CGAGGGCCATCATAGAACAG \\
IL-1 $\beta$ & AAAGCTCTCCACCTCAATGG & AGGCCACAGGTATTTTGTCG \\
TNF $\alpha$ & CCCCAAAGGGATGAGAAGTT & TGGGCTACAGGCTTGTCACT \\
CCL3 & CTGCCCTTGCTGTTCTTCTC & GTGGAATCTTCCGGCTGTAG \\
CCL4 & ATGAAGCTCTGCGTGTCTGC & GCCGGGAGGTGTAAGAGAAA \\
TLR4 & TGTTCTTCTCCTGCCTGACA & TGTCATCAGGGACTTTGCTG \\
\hline
\end{tabular}

GAPDH, glyceraldehyde-3-phosphate dehydrogenase. 


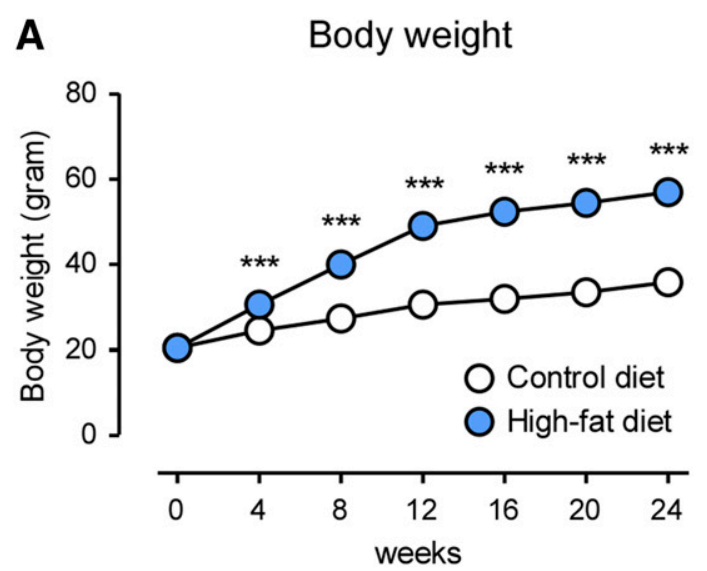

C

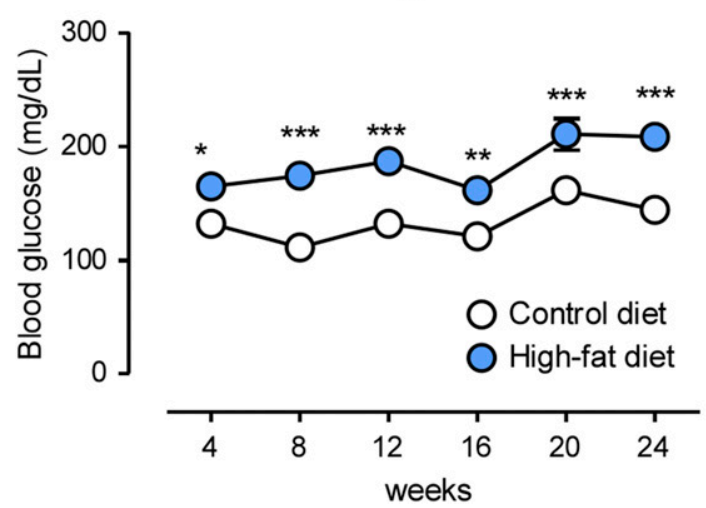

B

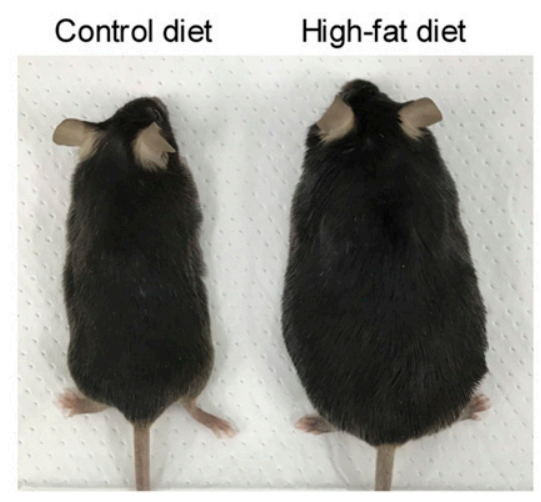

D

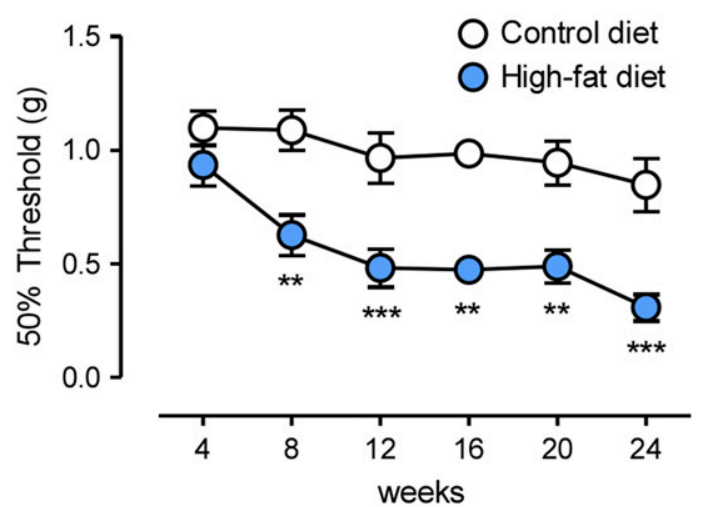

Fig. 1. Development of neuropathic pain associated with T2DM. Mice were fed an HFD or CD for 24 weeks, and each parameter was evaluated at the indicated periods. Time courses of body weight (A) and blood glucose levels (C) during HFD or CD feeding are shown. (B) Representative pictures at 16 weeks of HFD or CD feeding. (D) Reduction of the 50\% paw withdrawal mechanical threshold was assessed using the von Frey test. Data are presented as the mean \pm S.E.M.; $n=5-10 . * * * P<0.001 ; * * P<0.01 ; * P<0.05$ vs. control diet.

17 weeks of HFD feeding were decreased by perineural treatment with Mac1-Sap (250 ng), as confirmed by RT-qPCR, supporting the ablation of macrophages (Fig. 4A). In addition, the mRNA expression levels of IL- $1 \beta$ ( $t$ test, $P=0.0434 ; n=6)$, CCL3 ( $t$ test, $P=0.0356 ; n=6$ ), and CCL4 ( $t$ test, $P=0.0318$; $n=6$ ) after HFD feeding were also decreased after Mac1-Sap treatment, suggesting that inflammatory macrophages play a key role in neuroinflammation in the SCN after HFD feeding (Fig. 4B). The expression levels of these molecules in the SCN after CD feeding were slightly decreased by Mac1-Sap treatment, but those were not significant (Supplemental Fig. 2).

To determine a direct relationship between macrophage accumulation in the SCN and mechanical allodynia, macrophages were inoculated in the tissue surrounding the SCN. Based on the upregulation of toll-like receptor 4 (TLR4) mRNA in the SCN at 16 weeks of HFD feeding ( $t$ test, $P=0.0018 ; n=6$ ) (Supplemental Fig. 3), peritoneal macrophages collected from naive mice were activated by LPS, an exogenous ligand for TLR4, treatment in vitro. The mRNA expression levels of IL-1 $\beta$ ( $t$ test, $P=0.0004 ; n=3)$, TNF $\alpha$ ( $t$ test, $P=0.0055 ; n=3)$, CCL3 ( $t$ test, $P=0.0026 ; n=3$ ), and CCL4 ( $t$ test, $P=0.0009$; $n=3$ ) were upregulated at 3 hours after LPS (500 ng/ml) treatment compared with vehicle treatment (Fig. 5A). Subsequently, activated macrophages were perineurally administered to naive mice. The mechanical pain threshold 3 days after the inoculation of LPS-treated macrophages was significantly lower than after vehicle-treated macrophage injection ( $t$ test, $P<0.001 ; n=8-9$ ) (Fig. 5B).

Finally, we investigated whether pharmacological inhibition of macrophages can relieve HFD-induced mechanical allodynia. Consistent with our previous reports showing that the $\alpha 4 \beta 2$ subtype of $\mathrm{nAChR}$ is a negative regulator of inflammatory macrophages underlying neuropathic pain, the $\alpha 4$ subunit of $\mathrm{nAChR}$ was located on accumulated $\mathrm{F} 4 / 80^{+}$ macrophages in the SCN after HFD feeding (Fig. 6A). TC-2559, a potent $\alpha 4 \beta 2 \mathrm{nAChR}$ agonist, was perineurally or systemically (subcutaneously) administered once a day for 4 days at 16 weeks of HFD feeding. The perineural administration of TC-2559 (20 nmol) significantly improved HFD-induced mechanical allodynia $[\mathrm{F}(4,75)=25.86$, $P<0.0001$; Bonferroni's test, $P<0.01$ ], and it was antagonized by the coadministration of $\mathrm{DH} \beta \mathrm{E}$ ( $40 \mathrm{nmol}$ ), an $\alpha 4 \beta 2 \mathrm{nAChR}$ antagonist (Fig. 6B). The relieving effects of perineural administration of TC-2559 were observed in a dose-dependent manner (20 nmol; $t$ test, $P=0.0008 ; n=5$ ) (Fig. 6C). Moreover, subcutaneous administration of TC-2559 (10 $\mathrm{mg} / \mathrm{kg})$ also relieved HFD-induced mechanical allodynia $(\mathrm{F}(3,75)=38.20$, $P<0.0001$; Bonferroni's test, $P<0.01$ ) (Fig. 6D). These relieving effects of TC-2559 (by perineural and subcutaneous administration) persisted for more than 1 week even after drug 


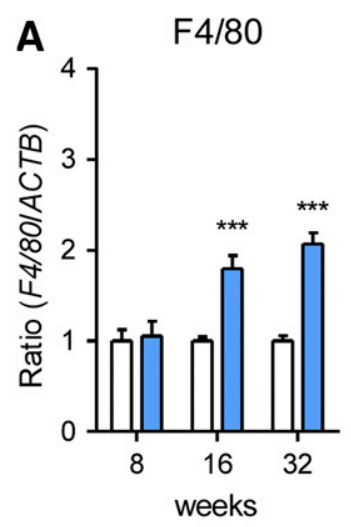

B

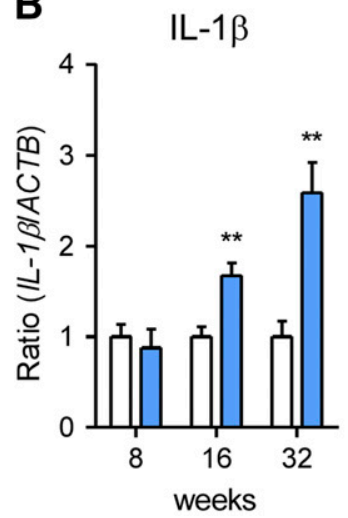

CD11b

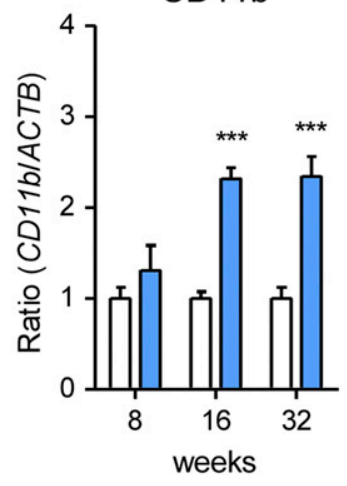

TNF $\alpha$

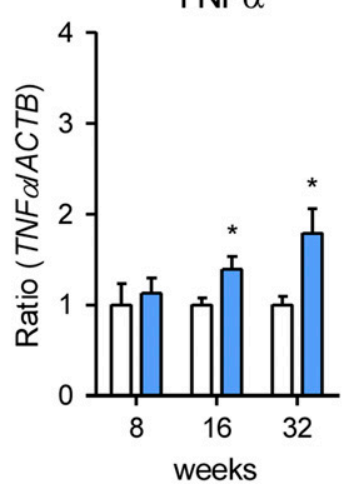

CD68

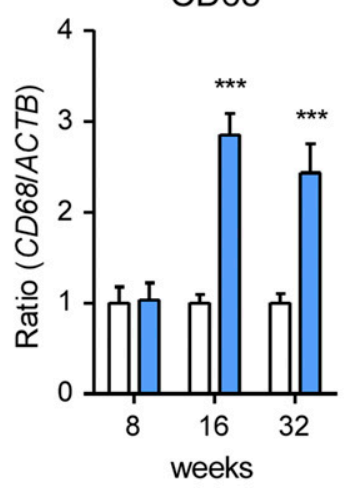

CCL3

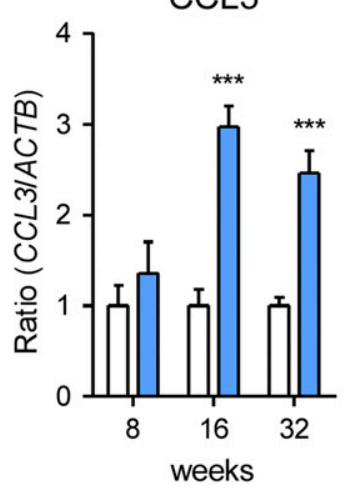

IRF5

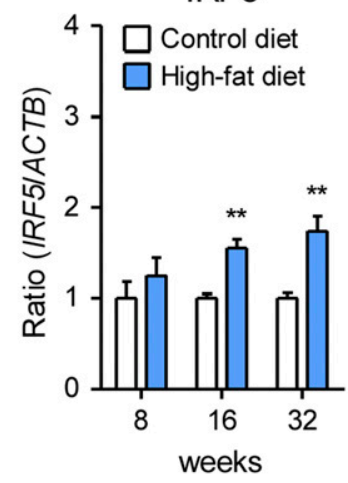

CCL4

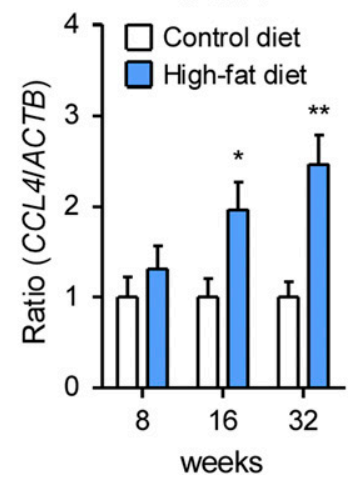

Fig. 2. Upregulation of macrophage markers and inflammatory mediators in the SCNs. Mice were fed an HFD or CD for 32 weeks, and the SCNs were collected at indicated periods. The expression of macrophage marker (F4/80, CD11b, CD68, and IRF5) (A) and inflammatory mediator (IL-1 $\beta$, $\mathrm{TNF} \alpha$, CCL3, and CCL4) (B) mRNA was analyzed by RT-qPCR. Data are presented as the mean \pm S.E.M.; $n=5-6$. ${ }^{* * *} P<0.001 ; * * P<0.01$; $* P<0.05$ vs. control diet.

cessation. TC-2559 did not affect the mechanical pain threshold in the CD group.

\section{Discussion}

The present study provides three novel findings suggesting that inflammatory macrophages in the SCN facilitate neuropathic pain associated with T2DM. First, the accumulation of inflammatory macrophages contributes to long-lasting neuroinflammation in the SCN after HFD feeding. Second, either the ablation of macrophages by cell-specific toxins or the pharmacological inhibition of inflammatory macrophages via $\alpha 4 \beta 2 \mathrm{nAChR}$ ligand improved HFD-induced mechanical allodynia. Third, inoculation of inflammatory macrophages in the tissue surrounding the SCN elicited mechanical allodynia.

Macrophages, a key component of innate immunity, play critical roles in the regulation of inflammatory responses and in host defense against pathogens (Gordon and Taylor, 2005; Murray and Wynn, 2011; Wynn et al., 2013; Okabe and Medzhitov, 2016). Upon tissue damage or infection, circulating monocytes are generally recruited to the site of damage by chemokine signaling (Murray and Wynn, 2011; Shi and Pamer, 2011; Wynn et al., 2013). Thereafter, monocytes differentiate into macrophages that orchestrate inflammatory responses to repair damaged tissue and resolve the excessive inflammation (Prame Kumar et al., 2018). On the other hand, when nervous systems are damaged, cytokines, chemokines, and damage-associated molecular patterns recruit macrophages into the injured nerves, which elicit nonresolving neuroinflammation (Kiguchi et al., 2017a). Macrophages are not the only cells to accumulate in damaged nerves, as neutrophils and lymphocytes also accumulate there (Perkins and Tracey, 2000; Moalem et al., 2004; Morin et al., 2007); however, macrophages account for the largest population at the middle/late phase after nerve injury (Kiguchi et al., 2017a). This suggests that macrophages may play a pivotal role in the regulation of neuroinflammation and pathologic pain. Notably, the ablation or inhibition of macrophages results in the prevention of experimental neuropathic pain in rodents (Liu et al., 2010; Echeverry et al., 2013; Kiguchi et al., 2014; Kobayashi et al., 2015). Macrophages are often polarized toward functionally distinct phenotypes to regulate complicated inflammatory processes (Ricardo et al., 2008; Murray and Wynn, 2011; Wynn et al., 2013). Inflammatory (M1) macrophages are characterized by higher expression of inflammatory cytokines and chemokines (i.e., IL-1 $\beta, \mathrm{TNF} \alpha, \mathrm{CCL} 3$, and CCL4) that exacerbate inflammation (Sica and Mantovani, 2012; Wynn and Vannella, 2016; Murray, 2017). In contrast, suppressive (M2) macrophages produce anti-inflammatory cytokines such as IL-10, which suppress and resolve inflammation (Mosser, 2003; Sica and Mantovani, 2012; Wynn and Vannella, 2016; Murray, 2017). Since the balance of macrophage polarization leans to the M1 state in injured nerves, neuroinflammation persists long term and is directly related to chronic pain 
A

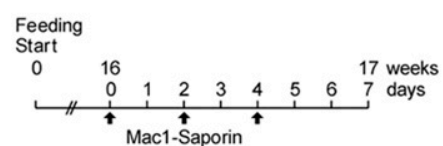

O CD / Saporin

$\checkmark \mathrm{CD} / \mathrm{Mac} 1-\mathrm{Sap}$

O HFD / Saporin

$\checkmark$ HFD / Mac1-Sap

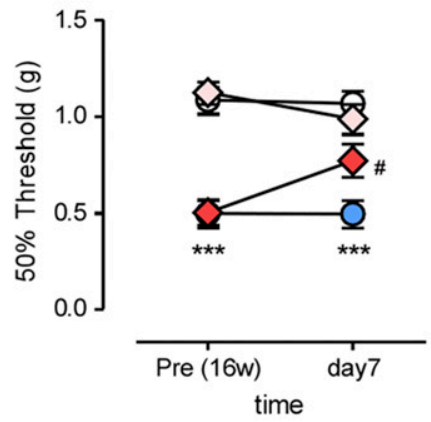

B

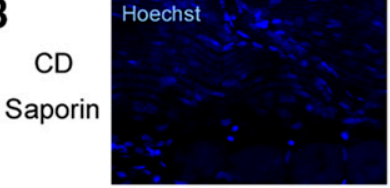

CD

Mac1Sap
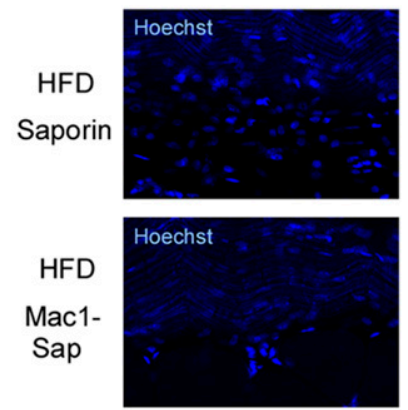

Number of macrophages

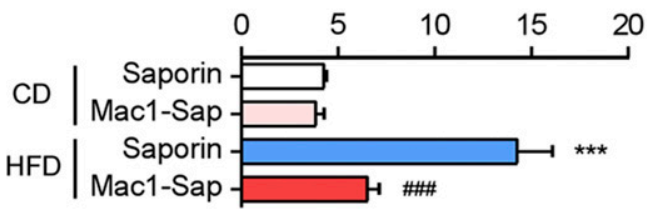

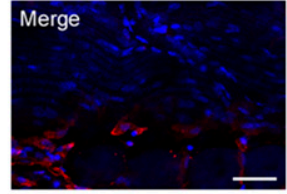

Merge
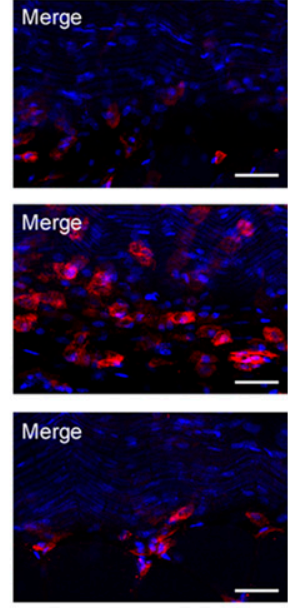

Fig. 3. Prevention of HFD-induced mechanical allodynia by the depletion of peripheral macrophages. Mice were fed an HFD or CD, and Mac1-Sap $(250 \mathrm{ng}$ ) or control saporin $(250 \mathrm{ng})$ was administered three times according to the marked schedule. (A) The $50 \%$ paw-withdrawal mechanical threshold was assessed using the von Frey test. (B) Representative micrographs and mean number of F4/80 macrophages in the SCNs on day 7 analyzed by immunohistochemistry are shown. Scale bar, $20 \mu \mathrm{m}$. Data are presented as the mean \pm S.E.M.; $n=4-11 . * * * P<0.001 \mathrm{vs}$. CD/saporin. \#P<0.05; $\# \#$ \# $<0.001$ vs. HFD/saporin.

(Kiguchi et al., 2015a, 2017b). We have demonstrated that the phenotypic shift of M1 macrophages toward M2 macrophages in injured nerves can relieve neuropathic pain (Kiguchi et al., 2015a, 2017b), supporting the notion that inflammatory macrophages are a fundamental component of neuropathic pain.

Inflammatory macrophages predominantly produce typical inflammatory cytokines (IL- $1 \beta$ and $\mathrm{TNF} \alpha$ ) and chemokines (CCL3 and CCL4), and those molecules are well known as crucial mediators in various inflammatory diseases (Sica and Mantovani, 2012). Moreover, the inhibition of such molecules effectively improved neuropathic pain after nerve injury in rodents, indicating that cytokines and chemokines may also contribute to the pathogenesis of neuropathic pain (Scholz and Woolf, 2007; Kiguchi et al., 2010; Saika et al., 2012). Under a neuroinflammatory state, inflammatory macrophages interact with the surrounding cells through a cytokine-chemokine network (Gouwy et al., 2005; Becher et al., 2017). Given that these cytokines and chemokines directly sensitize primary sensory neurons through the sensitization of nociceptors, these mediators may contribute significantly to the peripheral regulation of neuropathic pain. Here we show that not only common macrophage markers (e.g., F4/80 and CD11b) but also IRF5 and TLR4, key inducers for M1 dominant molecules (IL-1 $\beta, \mathrm{TNF} \alpha, \mathrm{CCL} 3$, and CCL4), are upregulated in the SCN after HFD feeding, indicating that accumulated macrophages polarize to the M1 state. In addition, M1 dominant markers were also upregulated in parallel with the increase of macrophage markers in the SCN, and the ablation of macrophages by perineural administration of Mac1-Sap significantly suppressed the upregulation of M1 dominant molecules and improved established mechanical allodynia after HFD feeding. It is important that plasma concentration of $\mathrm{TNF} \alpha$ and the mRNA expression level of TLR4 in monocytes are significantly higher in patients with diabetic peripheral neuropathy compared with healthy volunteers (Zhu et al., 2017), supporting our results. These lines of evidence suggest that macrophagedriven neuroinflammation in the SCN mediates T2DM-related neuropathic pain. Interestingly, these neuroinflammatory events were observed in the SCN, but not in other peripheral nerves such as trigeminal nerves. Although the regionspecific mechanisms eliciting HFD-induced neuroinflammation are still unclear, this fact could correlate to the incidence of peripheral neuropathy in the feet of patients with T2DM (Gonçalves et al., 2017).

Growing evidence indicates that $\mathrm{nAChR}$ can be a negative regulator of inflammatory macrophages underlying intractable inflammatory diseases. We have previously demonstrated that the $\alpha 4 \beta 2$ subtype of $\mathrm{nAChR}$ mainly exerts anti-inflammatory effects on accumulated macrophages in the SCN after nerve injury (Saika et al., 2015; Kiguchi et al., 2018), whereas the $\alpha 7$ subtype has been a focus in other inflammatory diseases (Wang et al., 2003; Kox et al., 2011; Patel et al., 2017). Indeed, perineural administration of 

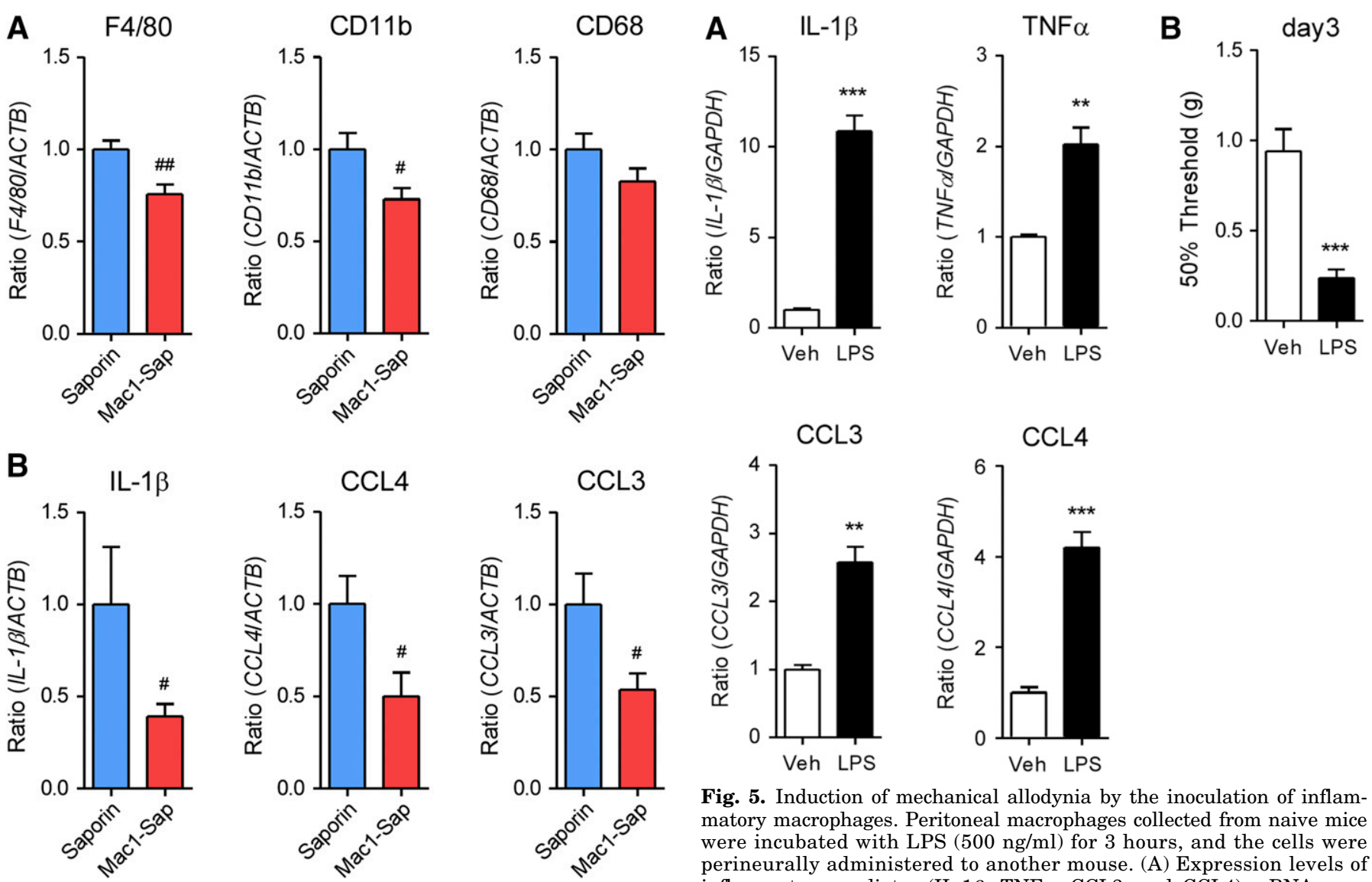

Fig. 4. Reduction of macrophage markers and inflammatory mediators in the SCN by the ablation of peripheral macrophages. Mice were fed an HFD, and Mac1-Sap (250 ng) or control saporin (250 ng) was administered three times, every 2 days. Expression levels of macrophage marker (F4/80, CD11b, and CD68) (A) and inflammatory mediator (IL-1 $\beta$, CCL3, and CCL4) (B) mRNA was analyzed by RT-qPCR. Data are presented as the mean \pm S.E.M.; $n=5-6$. \#\#P $<0.01$; \#P $<0.05$ vs. HFD/saporin.

nicotine or TC-2559 significantly prevented neuropathic pain caused by SCN injury in mice (Saika et al., 2015; Kiguchi et al., 2018). We also revealed that activation of $\alpha 4 \beta 2 \mathrm{nAChR}$ decreased under high expression of M1 dominant markers in LPS-stimulated macrophages in vitro, and such effects were mediated, at least in part, by inhibition of the janus kinase 2-signal transducer and activator of transcription 3 pathway, a key transcription factor for inflammatory processes (Kiguchi et al., 2015b). Hence, we sought to investigate the effects of TC-2559 on HFD-induced mechanical allodynia as a pharmacological inhibitor of inflammatory macrophages. Either perineural or systemic administration of TC-2559 effectively improved HFDinduced mechanical allodynia, and this improvement persisted long term, even after the cessation of administration, indicating that macrophages might contribute to the maintenance of T2DM-related neuropathic pain. So far, a direct relationship between inflammatory macrophages and mechanical allodynia has not fully determined. Inoculation of LPS-activated peritoneal macrophages into tissue surrounding the SCN showing M1 phenotype elicited mechanical allodynia, which is consistent with the results of macrophage ablation or inhibition. These results further support the premise that inflammatory macrophages

Fig. 5. Induction of mechanical allodynia by the inoculation of inflammatory macrophages. Peritoneal macrophages collected from naive mice were incubated with LPS $(500 \mathrm{ng} / \mathrm{ml})$ for 3 hours, and the cells were perineurally administered to another mouse. (A) Expression levels of inflammatory mediator (IL-1 $\beta, \mathrm{TNF} \alpha, \mathrm{CCL} 3$, and CCL4) mRNA were analyzed by RT-qPCR. (B) The 50\% paw-withdrawal mechanical threshold on day 3 after inoculation was assessed using the von Frey test. Data are presented as the mean \pm S.E.M.; $n=3-10 .{ }^{* * *} P<0.001 ; * * P<0.01$ vs. vehicle (Veh). GAPDH, glyceraldehyde-3-phosphate dehydrogenase.

directly contribute to mechanical allodynia, and that inflammatory macrophages are important for the pathogenesis of neuropathic pain caused not only by nerve injury but also by T2DM.

Unlike type 1 diabetes mellitus, the pathophysiology of T2DM is closely related to obesity in patients, and it is well known that adipose tissues exacerbate insulin resistance and hyperglycemia (Kahn and Flier, 2000; Martyn et al., 2008). Therefore, the HFD feeding model, which shows not only hyperglycemia but also obesity, is commonly used as an experimental mouse model of T2DM. In our results, the induction of mechanical allodynia did not completely correlate to the time course of macrophage accumulation. Nevertheless, the induction period is consistent with other reports using similar HFD feeding models (Jayaraj et al., 2018). These lines of evidence suggest that the molecular mechanisms of mechanical allodynia in the induction phase might be different from those in the maintenance phase. Based on our functional study, inflammatory macrophages might play a significant role in the maintenance phase of HFD-induced allodynia. Obesity is defined by the activation and hypertrophy of adipocytes consisting of a large amount of fat, and it is facilitated by chronic inflammation (Weisberg et al., 2003). In particular, cross talk between adipocytes and macrophages through the cytokine-chemokine network has been extensively investigated (Wellen and Hotamisligil, 2003; Xu et al., 2003; Nishimura et al., 2008; 
A
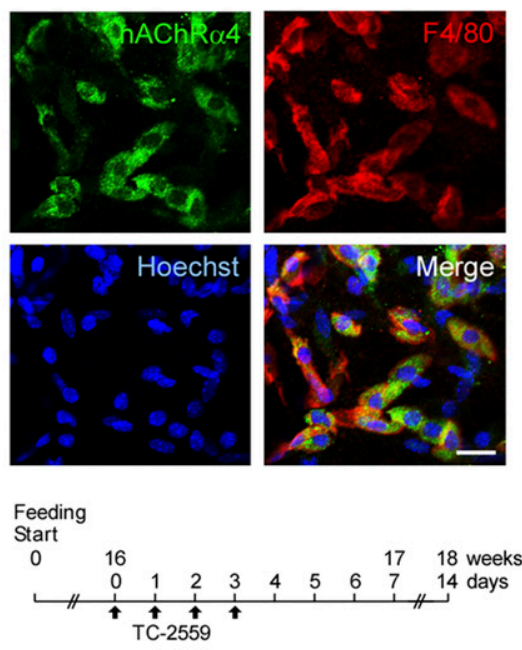

C

Perineural

$\nabla \mathrm{CD} / \mathrm{TC}-2559(2 \mathrm{nmol})$

$\nabla \mathrm{HFD} / \mathrm{TC}-2559(0.2 \mathrm{nmol})$

$\nabla \mathrm{HFD} / \mathrm{TC}-2559(2 \mathrm{nmol})$

$\nabla$ HFD / TC-2559 (20 nmol)

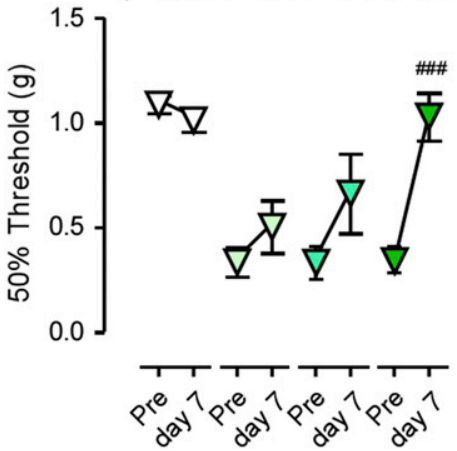

B

\section{Perineural}

$\mathrm{O} \mathrm{CD} /$ Vehicle

$\nabla$ CD / TC-2559

$\mathrm{OHFD} /$ Vehicle

$\nabla$ HFD / TC-2559

HFD / TC-2559+DH $\beta E$

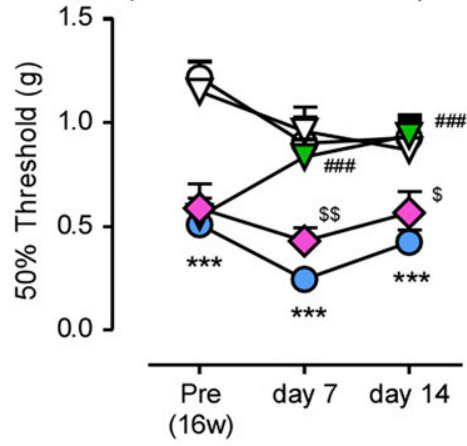

D

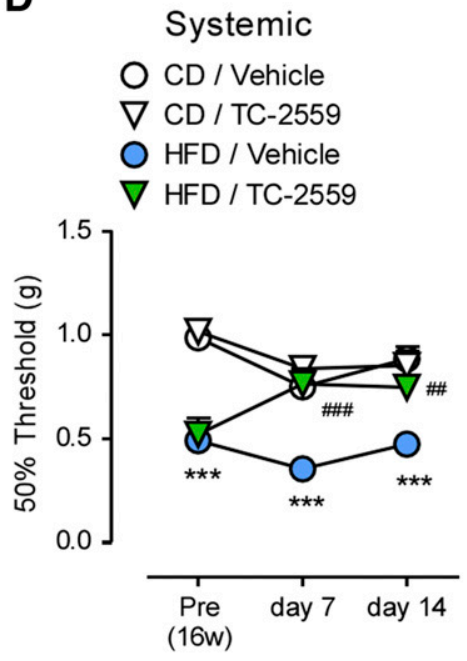

Fig. 6. Improvement of HFD-induced mechanical allodynia by treatment with $\alpha 4 \beta 2 \mathrm{nAChR}$ agonist. Mice were fed an HFD or CD, and the SCNs were collected. (A) Localization of the nAChR $\alpha 4$ subunit on $\mathrm{F} 4 / 80^{+}$macrophages at 16 weeks of HFD feeding, analyzed by immunohistochemistry. Scale bar, $20 \mu \mathrm{m}$. Effects of perineurally [20 nmol (B), $0.2-20 \mathrm{nmol}(\mathrm{C})]$ or systemically [10 mg/kg (D)] administered TC-2559 on HFD-induced mechanical allodynia. TC-2559 and $\mathrm{DH} \beta \mathrm{E}(40 \mathrm{nmol})$ were administered four times according to the discussed schedules, and a 50\% paw-withdrawal mechanical threshold was assessed using the von Frey test. Data are presented as the mean \pm S.E.M.; $n=6-8 .{ }^{* * *} P<0.001$ vs. CD/vehicle. \#\#\#P<0.001; $\# \# P<0.01$ vs. $\mathrm{HFD} /$ vehicle or HFD/Pre. $\$ \$ P<0.01 ; \$ P<0.05$ vs. HFD/TC-2559.
Schäffler and Schölmerich, 2010). In addition, adipocytes produce typical adipokines (i.e., leptin and adiponectin) that have several physiologic and pathologic functions (Falcão-Pires et al., 2012). Accumulating evidence suggests that adipokines act as a key regulator of inflammation and significantly participate in the pathophysiology of inflammatory diseases (Fantuzzi, 2005; Ouchi et al., 2011). Importantly, adipokines including leptin contribute to pathologic pain by the direct activation of macrophages and pain-processing neurons (Maeda et al., 2009). In this T2DM model, obesity-driven actions might be important for regulating both hyperglycemia and neuropathic pain. However, this is not clear and should be determined in future studies. Recent reports demonstrate that sensory processing is altered by HFD feeding and that excessive innervation of sensory neurons to the skin may underlie abnormal hypersensitivity (Jayaraj et al., 2018). In terms of macrophagedriven neuroinflammatory events in the peripheral nerves, it is worth understanding the relationship between these phenomena and inflammatory macrophages.

In conclusion, inflammatory macrophages accumulated in the SCNs mediate the pathophysiology of neuropathic pain associated with T2DM. Neuroinflammation driven by inflammatory macrophages through a cytokine-chemokine network plays a crucial role in neuropathic pain not only in nerve injury, but also in T2DM; this is supported by the fact that the ablation or inhibition of accumulated inflammatory macrophages significantly improved neuropathic pain. Moreover, the ability of macrophages to induce neuropathic pain is also confirmed by evidence showing that the inoculation of activated inflammatory macrophages elicits mechanical allodynia under normal conditions. Collectively, the functional significance of inflammatory macrophages for the peripheral regulation of neuropathic pain is clearly determined and such events are commonly observed not only in nerve injury-related neuropathic pain, but also in T2DM-related neuropathic pain. We propose that pharmacological inhibition of macrophages themselves or macrophage-derived cytokines and chemokines could be a potential novel candidate in pharmacotherapy against several kinds of neuropathic pain.

\section{Acknowledgments}

We thank Prof.Takehiko Maeda (Niigata University of Pharmacy and Applied Life Sciences, Niigata, Japan) and Prof.Toshio Shimokawa 
(Wakayama Medical University) for data analysis and suggestions. We also thank Editage for editing this manuscript.

\section{Authorship Contributions}

Participated in research design: Kiguchi and Kishioka.

Conducted experiments: Saika, Kiguchi, Matsuzaki, and Kobayashi.

Performed data analysis: Saika, Kiguchi, and Matsuzaki.

Wrote or contributed to the writing of the manuscript: Saika, Kiguchi, and Kishioka.

\section{References}

Austin PJ and Moalem-Taylor G (2010) The neuro-immune balance in neuropathic pain: involvement of inflammatory immune cells, immune-like glial cells and cytokines. J Neuroimmunol 229:26-50

Baron R, Tölle TR, Gockel U, Brosz M, and Freynhagen R (2009) A cross-sectional cohort survey in 2100 patients with painful diabetic neuropathy and postherpetic neuralgia: differences in demographic data and sensory symptoms. Pain 146: $34-40$

Barrett AM, Lucero MA, Le T, Robinson RL, Dworkin RH, and Chappell AS (2007) Epidemiology, public health burden, and treatment of diabetic peripheral neuropathic pain: a review. Pain Med 8 (Suppl 2):S50-S62.

Becher B, Spath S, and Goverman J (2017) Cytokine networks in neuroinflammation. Nat Rev Immunol 17:49-59.

Boulton AJ, Vinik AI, Arezzo JC, Bril V, Feldman EL, Freeman R, Malik RA, Maser RE, Sosenko JM, and Ziegler D; American Diabetes Association (2005) Diabetic neuropathies: a statement by the American Diabetes Association. Diabetes Care 28:956-962.

Calvo M, Dawes JM, and Bennett DL (2012) The role of the immune system in the generation of neuropathic pain. Lancet Neurol 11:629-642.

Chaplan SR, Bach FW, Pogrel JW, Chung JM, and Yaksh TL (1994) Quantitative assessment of tactile allodynia in the rat paw. J Neurosci Methods 53:55-63.

Drouin-Ouellet J and Cicchetti F (2012) Inflammation and neurodegeneration: the story 'retolled'. Trends Pharmacol Sci 33:542-551.

Echeverry S, Wu Y, and Zhang J (2013) Selectively reducing cytokine/chemokine expressing macrophages in injured nerves impairs the development of neuropathic pain. Exp Neurol 240:205-218.

Falcão-Pires I, Castro-Chaves P, Miranda-Silva D, Lourenço AP, and Leite-Moreira $\mathrm{AF}$ (2012) Physiological, pathological and potential therapeutic roles of adipokines. Drug Discov Today 17:880-889.

Fantuzzi G (2005) Adipose tissue, adipokines, and inflammation. J Allergy Clin Immunol 115:911-919, quiz 920.

Gonçalves NP, Vægter CB, Andersen H, Østergaard L, Calcutt NA, and Jensen TS (2017) Schwann cell interactions with axons and microvessels in diabetic neuropathy. Nat Rev Neurol 13:135-147.

Gordon S and Taylor PR (2005) Monocyte and macrophage heterogeneity. Nat Rev Immunol 5:953-964.

Gouwy M, Struyf S, Proost P, and Van Damme J (2005) Synergy in cytokine and chemokine networks amplifies the inflammatory response. Cytokine Growth Factor Rev 16:561-580.

Heydemann A (2016) An overview of murine high fat diet as a model for type 2 diabetes mellitus. J Diabetes Res 2016:2902351.

$\mathrm{Hu} \mathrm{P}$ and McLachlan EM (2002) Macrophage and lymphocyte invasion of dorsal root ganglia after peripheral nerve lesions in the rat. Neuroscience 112:23-38.

Jayaraj ND, Bhattacharyya BJ, Belmadani AA, Ren D, Rathwell CA, Hackelberg S, Hopkins BE, Gupta HR, Miller RJ, and Menichella DM (2018) Reducing CXCR4mediated nociceptor hyperexcitability reverses painful diabetic neuropathy. J Clin Invest 128:2205-2225.

Kahn BB and Flier JS (2000) Obesity and insulin resistance. J Clin Invest 106: 473-481.

Kiguchi N, Kobayashi D, Saika F, Matsuzaki S, and Kishioka S (2017a) Pharmacological regulation of neuropathic pain driven by inflammatory macrophages. Int $J$ Mol Sci 18:E2296.

Kiguchi N, Kobayashi D, Saika F, Matsuzaki S, and Kishioka S (2018) Inhibition of peripheral macrophages by nicotinic acetylcholine receptor agonists suppresses spinal microglial activation and neuropathic pain in mice with peripheral nerve injury. $J$ Neuroinflammation 15:96.

Kiguchi N, Kobayashi Y, Kadowaki Y, Fukazawa Y, Saika F, and Kishioka S (2014) Vascular endothelial growth factor signaling in injured nerves underlies peripheral sensitization in neuropathic pain. J Neurochem 129:169-178.

Kiguchi N, Kobayashi Y, Saika F, Sakaguchi H, Maeda T, and Kishioka S (2015a) Peripheral interleukin-4 ameliorates inflammatory macrophage-dependent neuropathic pain. Pain 156:684-693.

Kiguchi N, Maeda T, Kobayashi Y, Fukazawa Y, and Kishioka S (2010) Macrophage inflammatory protein-1alpha mediates the development of neuropathic pain following peripheral nerve injury through interleukin-1beta up-regulation. Pain 149 $305-315$.

Kiguchi N, Saika F, Kobayashi Y, Ko MC, and Kishioka S (2015b) TC-2559, an $\alpha 4 \beta 2$ nicotinic acetylcholine receptor agonist, suppresses the expression of CCL3 and IL-1 $\beta$ through STAT3 inhibition in cultured murine macrophages. J Pharmacol Sci 128:83-86.

Kiguchi N, Sakaguchi H, Kadowaki Y, Saika F, Fukazawa Y, Matsuzaki S, and Kishioka S (2017b) Peripheral administration of interleukin-13 reverses inflammatory macrophage and tactile allodynia in mice with partial sciatic nerve ligation. J Pharmacol Sci 133:53-56.

Kobayashi Y, Kiguchi N, Fukazawa Y, Saika F, Maeda T, and Kishioka S (2015) Macrophage-T cell interactions mediate neuropathic pain through the glucocorticoid-induced tumor necrosis factor ligand system. J Biol Chem $\mathbf{2 9 0}$ 12603-12613.

Kox M, Pompe JC, Peters E, Vaneker M, van der Laak JW, van der Hoeven JG, Scheffer GJ, Hoedemaekers CW, and Pickkers P (2011) $\alpha 7$ nicotinic acetylcholine receptor agonist GTS-21 attenuates ventilator-induced tumour necrosis factor- $\alpha$ production and lung injury. $\mathrm{Br} J$ Anaesth 107:559-566.

Lee S and Zhang J (2012) Heterogeneity of macrophages in injured trigeminal nerves: cytokine/chemokine expressing vs. phagocytic macrophages. Brain Behav Immun 26:891-903.

Liu CC, Lu N, Cui Y, Yang T, Zhao ZQ, Xin WJ, and Liu XG (2010) Prevention of paclitaxel-induced allodynia by minocycline: effect on loss of peripheral nerve fibers and infiltration of macrophages in rats. Mol Pain 6:76.

Liu T, van Rooijen N, and Tracey DJ (2000) Depletion of macrophages reduces axonal degeneration and hyperalgesia following nerve injury. Pain 86:25-32.

Ma W and Quirion R (2006) Increased calcitonin gene-related peptide in neuroma and invading macrophages is involved in the up-regulation of interleukin- 6 and thermal hyperalgesia in a rat model of mononeuropathy. J Neurochem 98:180-192.

Maeda T, Kiguchi N, Kobayashi Y, Ikuta T, Ozaki M, and Kishioka S (2009) Leptin derived from adipocytes in injured peripheral nerves facilitates development of neuropathic pain via macrophage stimulation. Proc Natl Acad Sci USA 106: 13076-13081.

Martyn JA, Kaneki M, and Yasuhara S (2008) Obesity-induced insulin resistance and hyperglycemia: etiologic factors and molecular mechanisms. Anesthesiology 109: 137-148.

Menichella DM, Abdelhak B, Ren D, Shum A, Frietag C, and Miller RJ (2014) CXCR4 chemokine receptor signaling mediates pain in diabetic neuropathy. Mol Pain 10: 42

Mert T, Gunay I, Ocal I, Guzel AI, Inal TC, Sencar L, and Polat S (2009) Macrophage depletion delays progression of neuropathic pain in diabetic animals. Naunyn Schmiedebergs Arch Pharmacol 379:445-452.

Moalem G, Xu K, and Yu L (2004) T lymphocytes play a role in neuropathic pain following peripheral nerve injury in rats. Neuroscience 129:767-777.

Morin N, Owolabi SA, Harty MW, Papa EF, Tracy TF Jr, Shaw SK, Kim M, and Saab CY (2007) Neutrophils invade lumbar dorsal root ganglia after chronic constriction injury of the sciatic nerve. J Neuroimmunol 184:164-171.

Mosser DM (2003) The many faces of macrophage activation. J Leukoc Biol 73 209-212

Murray PJ (2017) Macrophage polarization. Annu Rev Physiol 79:541-566.

Murray PJ and Wynn TA (2011) Protective and pathogenic functions of macrophage subsets. Nat Rev Immunol 11:723-737.

Nishimura S, Manabe I, Nagasaki M, Seo K, Yamashita H, Hosoya Y, Ohsugi M, Tobe K, Kadowaki T, Nagai R, et al. (2008) In vivo imaging in mice reveals local cell dynamics and inflammation in obese adipose tissue. $J$ Clin Invest 118: $710-721$.

Okabe Y and Medzhitov R (2016) Tissue biology perspective on macrophages. Nat Immunol 17:9-17.

Ouchi N, Parker JL, Lugus JJ, and Walsh K (2011) Adipokines in inflammation and metabolic disease. Nat Rev Immunol 11:85-97.

Patel H, McIntire J, Ryan S, Dunah A, and Loring R (2017) Anti-inflammatory effects of astroglial $\alpha 7$ nicotinic acetylcholine receptors are mediated by inhibition of the $\mathrm{NF}-\kappa \mathrm{B}$ pathway and activation of the Nrf2 pathway. J Neuroinflammation 14:192.

Perkins NM and Tracey DJ (2000) Hyperalgesia due to nerve injury: role of neutrophils. Neuroscience 101:745-757.

Prame Kumar K, Nicholls AJ, and Wong CHY (2018) Partners in crime: neutrophils and monocytes/macrophages in inflammation and disease. Cell Tissue Res $\mathbf{3 7 1}$ 551-565.

Ricardo SD, van Goor H, and Eddy AA (2008) Macrophage diversity in renal injury and repair. $J$ Clin Invest 118:3522-3530.

Saika F, Kiguchi N, Kobayashi Y, Fukazawa Y, and Kishioka S (2012) CC-chemokine ligand 4 /macrophage inflammatory protein-1 $\beta$ participates in the induction of neuropathic pain after peripheral nerve injury. Eur J Pain 16:1271-1280.

Saika F, Kiguchi N, Kobayashi Y, and Kishioka S (2015) Peripheral alpha4beta2 nicotinic acetylcholine receptor signalling attenuates tactile allodynia and thermal hyperalgesia after nerve injury in mice. Acta Physiol (Oxf) 213:462-471.

Schäffler A and Schölmerich J (2010) Innate immunity and adipose tissue biology. Trends Immunol 31:228-235.

Scholz J and Woolf CJ (2007) The neuropathic pain triad: neurons, immune cells and glia. Nat Neurosci 10:1361-1368.

Shi C and Pamer EG (2011) Monocyte recruitment during infection and in flammation. Nat Rev Immunol 11:762-774.

Sica A and Mantovani A (2012) Macrophage plasticity and polarization: in vivo veritas. J Clin Invest 122:787-795.

Sommer C and Kress M (2004) Recent findings on how proinflammatory cytokines cause pain: peripheral mechanisms in inflammatory and neuropathic hyperalgesia Neurosci Lett 361:184-187.

Sudore RL, Karter AJ, Huang ES, Moffet HH, Laiteerapong N, Schenker Y, Adams A Whitmer RA, Liu JY, Miao Y, et al. (2012) Symptom burden of adults with type 2 diabetes across the disease course: diabetes \& aging study. J Gen Intern Med $\mathbf{2 7}$ 1674-1681.

Thacker MA, Clark AK, Marchand F, and McMahon SB (2007) Pathophysiology of peripheral neuropathic pain: immune cells and molecules. Anesth Analg 105: 838-847.

Ubogu EE, Cossoy MB, and Ransohoff RM (2006) The expression and function of chemokines involved in CNS inflammation. Trends Pharmacol Sci 27:48-55.

Wang H, Yu M, Ochani M, Amella CA, Tanovic M, Susarla S, Li JH, Wang H, Yang H, Ulloa L, et al. (2003) Nicotinic acetylcholine receptor alpha7 subunit is an essential regulator of inflammation. Nature 421:384-388.

Weisberg SP, McCann D, Desai M, Rosenbaum M, Leibel RL, and Ferrante AW Jr (2003) Obesity is associated with macrophage accumulation in adipose tissue. $J$ Clin Invest 112:1796-1808. 
Wellen KE and Hotamisligil GS (2003) Obesity-induced inflammatory changes in adipose tissue. $J$ Clin Invest 112:1785-1788.

Wynn TA, Chawla A, and Pollard JW (2013) Macrophage biology in development, homeostasis and disease. Nature 496:445-455.

Wynn TA and Vannella KM (2016) Macrophages in tissue repair, regeneration, and fibrosis. Immunity 44:450-462.

Xu H, Barnes GT, Yang Q, Tan G, Yang D, Chou CJ, Sole J, Nichols A, Ross JS, Tartaglia LA, et al. (2003) Chronic inflammation in fat plays a crucial role in the development of obesity-related insulin resistance. J Clin Invest 112:1821-1830.
Zhu T, Meng Q, Ji J, Zhang L, and Lou X (2017) TLR4 and caveolin-1 in monocytes are associated with inflammatory conditions in diabetic neuropathy. Clin Transl Sci 10:178-184.

Address correspondence to: Dr. Norikazu Kiguchi, Wakayama Medical University, 811-1 Kimiidera, Wakayama City, Wakayama 641-0012, Japan. E-mail: kiguchi@wakayama-med.ac.jp 